\title{
\begin{tabular}{l} 
EXPERT \\
LEVIEWS \\
\hline
\end{tabular}
}

\section{Updates on the biology and management of dyskeratosis congenita and related telomere biology disorders}

Expert Rev. Hematol. 6(3), 327-337 (2013)

\section{Bari J Ballew \& Sharon A Savage*}

Division of Cancer Epidemiology and Genetics, National Cancer Institute, 6120 Executive Blvd. EPS 7018,

Rockville, MD 20892, USA *Author for correspondence: Tel.: + 13014965785 savagesh@mail.nih.gov

Dyskeratosis congenita (DC) is a cancer-prone inherited bone marrow failure syndrome caused by aberrant telomere biology. The mucocutaneous triad of nail dysplasia, abnormal skin pigmentation and oral leukoplakia is diagnostic, but is not always present; DC can also be diagnosed by the presence of very short leukocyte telomeres. Patients with DC are at high risk of bone marrow failure, pulmonary fibrosis, liver disease, cancer and other medical problems. Germline mutations in one of nine genes associated with telomere maintenance are present in approximately $60 \%$ of patients. DC is one among the group of clinically and biologically related telomere biology disorders, including Hoyeraal-Hreidarsson syndrome, Revesz syndrome, Coats plus (also known as cranioretinal microangiopathy with calcifications and cysts) and subsets of aplastic anemia, pulmonary fibrosis, nonalcoholic and noninfectious liver disease and leukemia. The authors review the pathobiology that connects DC and the related telomere biology disorders, methods of diagnosis and management modalities.

KeYwords: aplastic anemia $\bullet$ cerebroretinal microangiopathy with calcification and cyst $\bullet$ Coats plus $\bullet$ congenital - dyskeratosis $\bullet$ Hoyeraal-Hreidarsson syndrome $\bullet$ inherited bone marrow failure syndrome $\bullet$ pulmonary fibrosis

- Revesz syndrome $\bullet$ shelterin $\bullet$ telomerase $\bullet$ telomere $\bullet$ telomere biology disorder

Dyskeratosis congenita (DC) is a rare cancerprone inherited bone marrow failure syndrome (IBMFS) caused by aberrant telomere biology $[1-4]$. It is characterized clinically by the presence of the diagnostic triad of dysplastic nails, lacy reticulated skin pigmentation and oral leukoplakia (FIGURe 1) [5]. The clinical phenotype is very broad and may also include pulmonary fibrosis, liver disease, avascular necrosis of the femur or humerus, dental and ophthalmologic abnormalities, and stenosis of the lacrimal ducts, esophagus and/or urethra. Notably, patients with DC are at very high risk of progressive bone marrow failure (BMF), myelodysplastic syndrome, acute myelogenous leukemia and solid tumors (usually squamous cell carcinoma of the head/neck or anogenital cancer) [6]. The unifying feature of this complex set of clinical problems is the presence of very short telomeres, the result of germline mutations in key telomere biology genes. Very short leukocyte telomeres (i.e., less than the first percentile for age) are diagnostic of DC [7]. A group of disorders caused by telomere defects, referred to as telomere biology disorders, is composed of a set of overlapping conditions including DC, Hoyeraal-Hreidarsson syndrome $(\mathrm{HH})$, Revesz syndrome (RS), Coats plus (also referred to as cranioretinal microangiopathy with calcifications and cysts [CRMCC]) [8,9], and some cases of apparently isolated aplastic anemia, idiopathic pulmonary fibrosis $[10,11]$, leukemia, as well as a subset of fibrotic liver disease [12]. Genetic and environmental modifiers of the phenotypes of DC and the related telomere biology disorders are not understood.

\section{Telomeres \& the end-replication problem}

Telomeres are specialized structures found at the ends of all eukaryotic chromosomes that are essential to preserving genome integrity. They are composed of tandem TTAGGG repeats [13] and range from two to more than 14 kilobases in length [14], although they are highly heterogeneous between chromosomes and between cells. Telomeres also have a single-strand DNA 

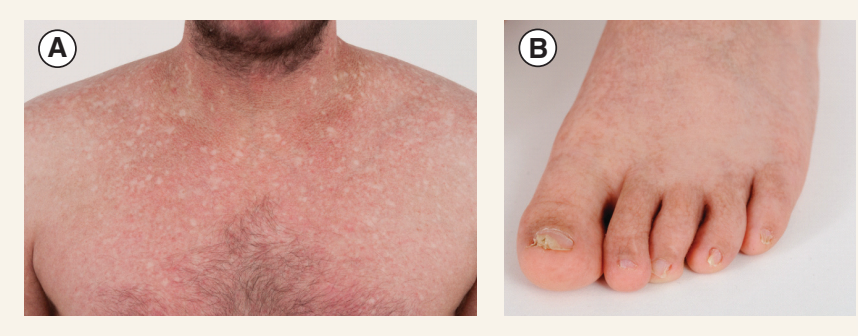

Figure 1. The diagnostic triad of dyskeratosis congenita. (A) Skin hyper- and hypo-pigmentation in a 32-year-old man; (B) toenail dystrophy in a 7-year-old boy; (C) oral leukoplakia on the tongue of 10-year-old girl.

overhang of approximately 200 nucleotides in length [15]. The duplex portion of the telomere and the telomeric overhang are critical for interactions with telomere-associated proteins.

After every cell division, there is attrition of the telomeric sequence at the ends of chromosomes as a consequence of semiconservative replication. This sequence loss has been referred to as the end-replication problem, and results in a process called replicative senescence, in which chromosomes get progressively shorter over many cell cycles $[16,17]$. The results of telomere shortening were first reported by Leonard Hayflick, who found that cells in culture could only survive a limited number of cell divisions [18]. When telomeres become critically short, the cell ceases to divide [19]. These critically short telomeres lose the protection of telomeric complexes, and can be recognized by the DNA damage repair machinery, resulting in cell-cycle arrest [20]. Attempts at repair of critically short telomeres can lead to genomic instability, a result of telomere-telomere fusions and chromosome breakage upon cell division [21-23]. The combination of genomic instability and subsequent somatic mutations that allow further cellular proliferation can result in oncogenesis [24].

To address the end-replication problem, telomerase, a specialized ribonucleoprotein, adds repetitive G-rich sequence on to telomere ends [25]. The telomerase enzyme is widely conserved, and consists of two essential components: the telomerase reverse transcriptase (TERT) and an internal RNA template that dictates the sequence of telomeric repeats, namely TERC [26]. These two subunits comprise the catalytic core; however, there are additional factors required for telomerase assembly and function in vivo. Dyskerin, encoded by $D K C 1$, associates with small nucleolar RNAs that contain H/ACA boxes, including TERC [2]. Dyskerin, along with GAR1, NHP2 and NOP10, is involved in ribosome biogenesis and rRNA pseudouridylation and localizes to both the nucleoli and Cajal bodies. These additional components have also been found to associate with TERC $[27,28]$ and appear to affect the stability, localization and regulation of the telomerase complex. TCAB1 (encoded by WRAP53) stably associates with the telomerase holoenzyme, and is necessary to direct TERC through Cajal bodies to the telomere [29]. The assembly chaperones NAF1 and SHQ1 [30] and the ATPases pontin (encoded by $R U V B L 1$ ) and reptin (encoded by $R U V B L 2$ ) are required for telomerase assembly, but are not present in the mature telomerase ribonucleoprotein complex [31,32].

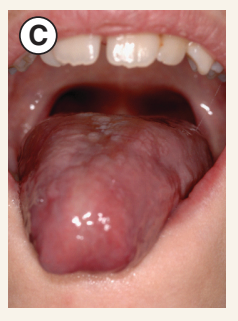

Most somatic cells have very little or no telomerase activity after the first few weeks of embryogenesis, and undergo replicative senescence during an organism's lifetime, while germline cells, stem cells, some immune cells, skin cells and the intestinal lining exhibit telomerase activity and maintain telomeres at a constant length [33-36]. Cells are very sensitive to changes in telomerase expression levels; mutations that affect telomerase levels have been discovered to cause human telomere biology disorders, including DC, aplastic anemia and idiopathic pulmonary fibrosis [37-39].

\section{DC: a disorder of aberrant telomere biology}

$\mathrm{DC}$ can be inherited in X-linked, autosomal dominant (AD) and autosomal recessive (AR) patterns, or can arise due to a de novo germline mutation. To date, a causative mutation in a DC-associated gene has been identified in approximately $60 \%$ of patients (Figure 2). Germline mutations in DKC1 were first identified as the cause of X-linked DC in 1998 [1]. The connection between DC and telomere length was made when dyskerin was shown to affect telomerase RNA. Primary fibroblasts and lymphoblasts from DC patients bearing DKC1 mutations exhibited low levels of telomerase RNA, reduced telomerase activity and short telomeres compared with normal controls [2].

The link between DC and telomere biology was supported by the subsequent discovery of hTERT or hTR (encoded by TERT and $T E R C$, respectively) mutations in patients with $\mathrm{AD}$ forms of DC $[3,4,40]$. The TERT mutations found in these patients are generally nonsynonymous coding mutations that lead to telomerase haploinsufficiency. TERC encodes the RNA template, which is required for the addition of telomeric nucleotide repeats by telomerase. In addition to mutations affecting the template region of TERC, mutations in the CCAAT box in the promoter region of TERC have been described [41]. Rarely, TERT can be mutated in AR forms of DC; bi-allelic mutations are associated with more severe disease and patients have dramatically reduced levels of telomerase. AR DC can also be the result of bi-allelic mutations in NOP10 or NHP2 (encoded by genes of the same names), both of which affect telomerase biogenesis.

Disruption of telomerase trafficking in the nucleus can result from germline mutations in TCAB1 (gene name WRAP53) [42]. Patients with compound heterozygous mutations in TCAB1 were reported to have features of classic DC. Their relatives who had a single mutant allele had normal telomere lengths, suggesting that bi-allelic mutations are required for this phenotype. Compound heterozygous mutations in patient cells prevented telomerase from localizing to Cajal bodies for assembly. This results in misdirection of telomerase RNA to the nucleoli and precludes telomerase from elongating telomeres.

Germline mutations in TIN2 (encoded by TINF2) are also responsible for AD DC. TIN2 is not directly involved in telomerase function; rather, it is part of the shelterin complex, a 
six-protein telomere-specific complex that protects telomeres and participates in length regulation. Mutations in the other shelterin components (TRF1, TRF2, POT1, TPP1 and RAP1) have not yet been found in DC. Causative TINF2 mutations cluster at the consensus site for HP1 1 . This association between TINF2 and HP1 $\gamma$ is required for sister telomere cohesion, thereby preventing sister telomere loss [43].

Compound heterozygous mutations in CTC1 were first reported as a cause of Coats plus and in the phenotypically similar disorder termed CRMCC. Patients with those mutations had short telomeres and features that phenotypically overlapped with DC $[8,9,44]$. Mutations in CTC1 were subsequently demonstrated to cause AR DC $[45,46]$. Patient telomere length in CTC1-associated DC was not as short as in DC of other causes, but still shorter than controls. CTC1 is part of the CST complex along with OBFC1 (STN1) and TEN1. The CST complex has both extratelomeric and telomeric roles; at the telomere, it cooperates with the shelterin complex to protect telomeres from degradation and aberrant recognition by DNA repair machinery.

Through exome sequencing, the authors recently discovered mutations in RTEL1 in three DC families with no mutations in the aforementioned eight genes [47]. RTEL1 regulates telomere length, may interact with PCNA (Proliferating Cell Nuclear Antigen) and also plays roles in DNA repair [48,49]. One family exhibited AR inheritance, another exhibited AD inheritance with likely genetic anticipation, and the third was likely AD, though the parental DNA was unavailable. Mutations in TERT have also been reported in both $\mathrm{AD}$ and $\mathrm{AR}$ inheritance patterns. An additional seven families with DC were recently reported to have AR RTEL1 mutations [50]. The molecular consequences of these mutations on RTEL1 function and telomere biology are still being elucidated. Early data suggest incorrect resolution of telomeric $\mathrm{T}$ loops may be the mechanism of aberrant telomere shortening [50].

Mutations in two additional genes have been reported in DC but their connection with telomere biology is less straightforward. An intraexonic splice variant in Apollo (encoded by DCLRE1B) was identified in a patient with clinical signs of $\mathrm{HH}$ but normal telomere lengths [51]. Apollo interacts with TRF2 and has some DNA repair functions, but its role in telomere biology is still being elucidated. Mutations in USB1 (formerly C16orf57), a gene without a known function, were found in individuals with DC-like symptoms but normal telomeres [52]. The patients with USB1 mutations had features of DC, but others with the same mutations had phenotypes consistent with Rothmund-Thomson syndrome and poikiloderma with neutropenia. This indicates some clinical overlap between DC

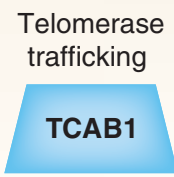

Telomerase enzyme complex
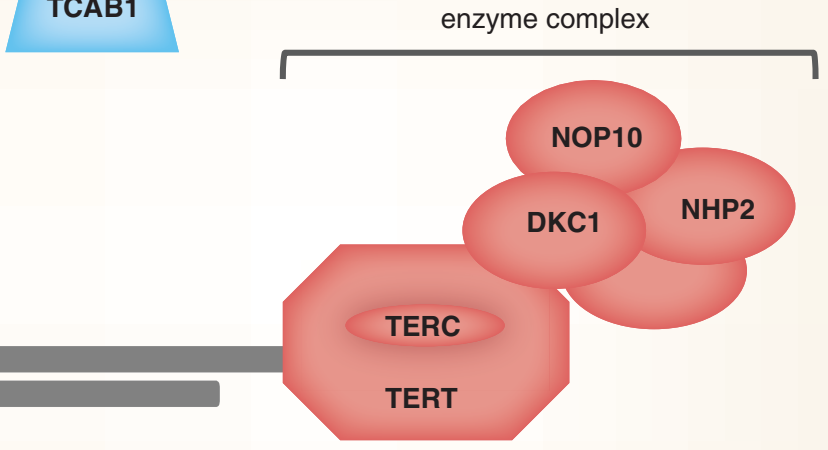

RTEL1

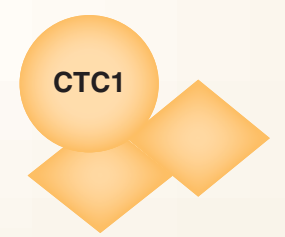

Telomere capping
Telomere stability and DNA helicase

\section{Telomere capping}

Figure 2. The telomere and functions of the proteins affected in dyskeratosis .

NHP2: NHP2 ribonucleoprotein (NHP2); NOP10: NOP10 ribonucleoprotein (NOP10)

associated protein 1 (WRAP53); TERC: Telomerase RNA component (TERC);

TERT: Telomerase (TERT); TIN2: TRF1-interacting nuclear factor 2 (TINF2).

and these other disorders, but there may be different underlying biological etiology. For both Apollo and USB1, the connection with telomere biology and DC is not yet understood.

\section{Genetic heterogeneity in DC}

Our understanding of inheritance patterns in DC is complicated by the presence of silent carriers, arising because of incomplete clinical penetrance of disease-associated mutations. Incomplete penetrance occurs in genetic disorders when a person with a disease-associated mutation does not develop the expected phenotype. This is possibly due to a combination of genetic, environmental and lifestyle factors. As more family members are tested for DC-associated mutations, more silent carriers are being recognized. Specifically, carriers of germline mutations in TERT, TERC and TINF2 with few symptoms consistent with DC have been identified because of the increased scrutiny brought about by the diagnosis of a family member. This occurs at least in part because the clinical signs and symptoms of DC can develop at different rates in different individuals, even within the same family. In one family with variable clinical penetrance, clinical symptoms of DC were not useful for a linkage scan, and the phenotype of very short telomeres was used instead to discover mutations in TINF2 as a cause of DC [53].

Genetic anticipation refers to a younger age of onset and increased severity of the symptoms of a disease over successive 
generations within a family. This was first reported in DC families with TERC mutations by Vulliamy et al. [54]. Subsequent reports included cases of telomerase haploinsufficiency due to TERT and TERC mutations: older generations may have adult-onset pulmonary fibrosis, but later generations with the same mutation tend to exhibit classic symptoms of DC and aplastic anemia in childhood [54-56]. A similar finding has been noted in a family with a TINF2 mutation [53]. It is also notable that in all of these reports the offspring have shorter telomeres than the parents. Because of the clinical heterogeneity, presence of silent carriers and genetic anticipation, all carriers of DC-associated mutations should be counseled regarding their own potential disease risk and that of their family members.

Genetic analysis of DC is made more complex by the recent identification of somatic mosaic reversion. This phenomenon has been reported in DC families where a germline TERC mutation identified in skin fibroblasts was spontaneously corrected by mitotic recombination in blood cells [57].

\section{Clinical features of DC}

The mucocutaneous features of DC were first described in the early 1900s. Subsequent reports led to its initial designation as Zinsser-Cole-Engman syndrome. It appears that the name was then changed to DC because of the mucocutaneous features and its congenital nature. Initial reports suggested primarily X-linked recessive inheritance. However, the first female case was reported in 1963 [58]. Subsequent case reports and small case series led to an appreciation of an expanded clinical phenotype, which included BMF and multiorgan system involvement. Variable expressivity - or a range of signs and symptoms in individuals with the same genetic condition - is now recognized in DC. Table 1 lists the clinical features that have been described in DC. In general, the classic triad of nail dystrophy, lacy reticular pigmentation of neck/upper chest and oral leukoplakia is diagnostic (FIGURE 1). However, the triad is not present in all affected individuals and can develop at variable ages.

Other clinical characteristics include eye abnormalities, dental abnormalities, esophageal stenosis, urethral stenosis, avascular necrosis of the femur/humerus, osteopenia, pulmonary fibrosis and nonalcoholic/noninfectious liver disease [38]. The clinical features found in patients with DC in the NCI cohort and those in the DC Registry are reported in TABLE 1 . In addition to these complications, patients are at high risk of bone marrow failure. The age of onset and disease progression vary widely, even within families. Detailed clinical study of the NCI DC cohort has led to improved quantification of DC-related complications and the characterization of new features. The presence of silent carriers can complicate the identification of an inheritance pattern; some affected family members may share an incompletely penetrant autosomal dominant allele with unaffected relatives.

Patients with DC are at increased risk of cancer, but their risk may vary depending on the genetic cause of their disease. Overall, patients with DC have an 11-fold increased risk of cancer compared with the general population [6]. More specifically, DC patients are at risk of cancers that tend to affect tissues with high turnover (skin, mucus membranes and bone marrow), including leukemia and squamous cell cancer of the head and neck or anogenital region. A study that evaluated data from the literature and the NCI DC cohort quantified the risks of certain cancers in individuals with DC. The actuarial risk of cancer in DC was $40 \%$ by age 50 years. The risks of certain cancers were substantially elevated compared with the general population. For example, the risk of tongue squamous cell cancer was 1154-fold greater in DC than in the general population and that of acute myelogenous leukemia was 195 -fold greater [6]. To date, one study has specifically evaluated the risk of cancer in patients with DC and specific germline mutations [59]. Six out of 30 patients with TERC mutations (20\%) and three out of 17 patients with $\mathrm{AD}$ TERT (17.6\%) mutations reported having had cancer compared with only one out of $56(1.8 \%)$ patients with TINF2 mutations. This may be because patients with TERT or TERC mutations tend to be older and the patients with TINF2 mutations have more severe BMF at a very young age. While both studies of cancer in DC are limited by relatively small sample sizes and the possibility of referral bias, they illustrate important connections between DC, telomere biology and cancer. Educating patients about potential cancer risks and self-exam of the oral cavity, head and neck, may lead to early detection of cancers.

Patients with DC are also at risk of dental and eye abnormalities. In total, $65 \%$ of DC patients have oral leukoplakia, one symptom of the diagnostic triad. In addition, DC patients may have a decreased root/crown ratio, taurodontism, increased dental caries, hypodontia, thin enamel structure, periodontitis, tooth loss or blunted roots [60]. A study of the ocular manifestations of DC in the NCI cohort reported that $28 \%$ of DC patients have an obstructed lacrimal drainage system [61]. Patients were also reported to have entropion and trichiasis, possibly secondary to epithelial abnormalities in the ocular skin and mucous membranes. RS, a severe subtype of DC discussed below, is also marked by bilateral exudative retinopathy.

Recent evidence from the NCI DC cohort suggests that patients with DC may have higher rates of neuropsychiatric disorders than the general population [62]. In total, $50 \%$ of children and $75 \%$ of adults with a DC or DC-like diagnosis had experienced a psychiatric disorder, compared with $25 \%$ of chronically ill children [62]. DC patients had evidence of psychiatric disorders (mood, anxiety, psychotic and adjustment disorders) or neurocognitive disorders (attention deficit hyperactivity disorder, intellectual disability, learning disabilities and pervasive developmental disorders).

\section{Diagnosing DC}

As mentioned earlier, the classic triad of nail dystrophy, lacy reticular pigmentation of neck/upper chest and oral leukoplakia is diagnostic, but is not present in all DC cases. Since the first description of shortened telomeres in cells derived from patients with DC [2], there has been interest in understanding telomere biology and its consequences on telomere length in DC. The connection between telomere biology and cancer has also led to numerous studies evaluating telomere length in surrogate tissues 
(i.e., blood or buccal cell DNA) as a biomarker of cancer risk. The development of a reproducible measurement of telomere length in leukocyte subsets by flow cytometry with in situ hybridization (flow FISH) led to the clinical test that is now used to diagnose DC. This method was shown to be highly sensitive and specific in differentiating patients with DC from their unaffected relatives, from patients with other IBMFS and from healthy controls [7,63,64]. Measurement of telomere length by other methods, including quantitative PCR and terminal restriction fragment Southern blots, are used in studying DC, but studies of the diagnostic sensitivity and specificity of these methods have not been conducted $[65,66]$.

\section{Genotype-phenotype correlations}

A limited number of genotype-phenotype correlation studies have been conducted in DC. Individuals with mutations in TINF2 or $D K C 1$ seem to have more complications and earlier bone marrow failure than other DC patients $[53,67]$. In addition, bi-allelic TERT mutations result in severe disease, while individuals with autosomal dominant TERC mutations have less clinically severe disease. In a genotype-phenotype study of the NCI DC cohort, DC patients with the shortest telomeres, measured by flow FISH in leukocyte subsets, generally had the most severe disease, earliest age of onset and earliest disease-related mortality [64]. Patients in that cohort without a known causative mutation, or with mutations in $D K C 1$, had the shortest telomeres and were most severely affected. The finding that the patients with an unknown genetic cause were more clinically severe could represent diagnostic or referral bias from the population evaluated in that study. The DC Registry reported that patients with DKC1 or TINF2 mutations were the most severely affected [59]. However, they did not find a relationship between telomere length, measured by quantitative PCR, and clinical severity. The differences between these two studies could be due to different telomere length measurement methods, clinical ascertainment biases and/or other unknown factors.

\section{Clinically \& biologically related telomere biology disorders}

The telomere biology disorders are a clinically and genetically heterogeneous group of disorders that include DC, HH, RS and Coats plus as well as others. In many instances, the genetic etiology overlaps and the clinical differences are a consequence of

\begin{tabular}{|c|c|c|}
\hline Feature & $\begin{array}{l}\text { DCR }(\%) \\
n=228\end{array}$ & $\begin{array}{l}\text { Literature cases } \\
(\%) ; n=550\end{array}$ \\
\hline DC triad (at least two of three features) & & 68 \\
\hline Dysplastic nails & 88 & 70 \\
\hline Reticulated skin pigmentation & 89 & 67 \\
\hline Oral leukoplakia & 78 & 47 \\
\hline Hematologic (cytopenias) & 86 & $50^{+}$ \\
\hline Epiphora & 31 & $29^{\ddagger}$ \\
\hline Dental abnormalities & 17 & 13 \\
\hline Short stature & 20 & 12 \\
\hline Intrauterine growth retardation or low birthweight & 8 & 9 \\
\hline Learning difficulty and/or developmental delay & 25 & 13 \\
\hline Microcephaly & 6 & 9 \\
\hline Cerebellar hypoplasia and/or ataxia & 7 & 5 \\
\hline Intracranial calcifications and/or leukomalacia & & 3 \\
\hline Reduced hearing & 1 & $<2^{\S}$ \\
\hline Pulmonary fibrosis & 20 & 7 \\
\hline Premature gray hair and/or early balding & 16 & 19 \\
\hline Esophageal narrowing, stricture or webs & 17 & 8 \\
\hline Enteropathy & 7 & \\
\hline Urethral stenosis/strictures/phimosis & 5 & 7 \\
\hline Undescended testes & 6 & 3 \\
\hline Osteopenia/osteoporosis/aseptic necrosis/scoliosis & 5 & 6 \\
\hline Cardiac anomalies & & 1 \\
\hline \multicolumn{3}{|c|}{$\begin{array}{l}\text { The DCR refers to the Dyskeratosis Congenita Registry, based in the UK [93,94]. } \\
\text { Literature cases refer to those compiled by Shimamura and Alter [77]. } \\
{ }^{\circ} \text { Approximately } 50 \% \text { of patients were estimated to have severe bone marrow failure by } 50 \text { years of age [6]. } \\
\text { Other findings, in addition to epiphora, were reported and include blepharitis, exudative retinopathy, } \\
\text { vascular retinopathy, strabismus, cataracts, absent eyelashes and ulcers. } \\
{ }^{\circledR} \text { Approximately } \% \% \text { of literature cases were reported to have rotated or low set ears or deafness [77]. } \\
\text { DC: Dyskeratosis congenita; DCR: Dyskeratosis Congenita Registry. }\end{array}$} \\
\hline
\end{tabular}

variable penetrance and/or expressivity and the presence of genetic anticipation of the phenotype. For all of the illnesses listed in the following subsections, a telomere biology disease should be considered during the diagnostic evaluation (FIgUre 3).

\section{HH syndrome}

Clinical features of what is now referred to as $\mathrm{HH}$ were first reported by Hoyeraal in 1970 and Hreidarsson in 1988 [68,69]. The connection between DC and HH was suggested due to overlapping clinical features, and was confirmed when mutations in $D K C 1$ were identified in both disorders [70]. AD mutations in TINF2 and AR mutations in TERT have also been reported as a cause of $\mathrm{HH}$. Patients with $\mathrm{HH}$ typically have features of DC, although the diagnostic triad may not be present in very young children. BMF can be severe at an early age. The presence of cerebellar hypoplasia in the setting of DC-related features is required for the diagnosis of $\mathrm{HH}$. Patients with $\mathrm{HH}$ often 


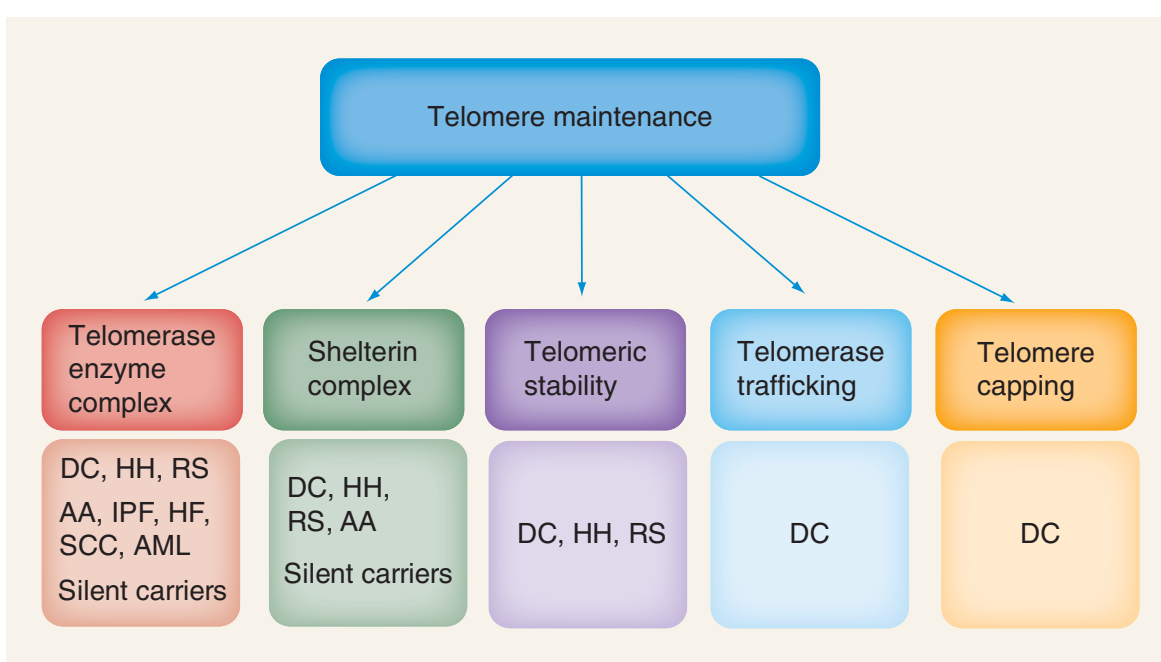

Figure 3. The telomere biology disorders are caused by aberrant function of different aspects of telomere maintenance. Short telomeres and the clinical features are a consequence of the molecular defect.

AA: Aplastic anemia; AML: Acute myeloid leukemia; DC: Dyskeratosis congenita;

HF: Hepatic fibrosis; HH: Hoyeraal-Hreidarsson syndrome; IPF: Idiopathic pulmonary

fibrosis; RS: Revesz syndrome; SCC: Early-onset head and neck or anogenital squamous cell cancer.

\section{Aplastic anemia}

Aplastic anemia is a heterogeneous BMF syndrome that can present at any age. Its etiology is multifactorial, and there are both inherited and acquired forms. The acquired form of aplastic anemia is typically immune-mediated and related to environmental exposures, infections or idiosyncratic reactions to medications. Patients with an inherited BMF syndrome can present with aplastic anemia as the primary feature. Primary presentation with aplastic anemia has also been reported in Fanconi anemia (FA) and ShwachmanDiamond syndrome, but may be more common in DC.

AD germline mutations in TERC and TERT have been reported to cause aplastic anemia in a subset of patients. Notably, a detailed review of the family history of patients with aplastic anemia may reveal disorders also seen in DC, such as pulmonary fibrosis, mild cytopenias, leuke- also have significant developmental delay, intrauterine growth retardation, immunodeficiency and nonspecific enteropathy.

\section{Revesz syndrome}

In 1992, Revesz et al. described a patient with bilateral exudative retinopathy at 6 months of age, who subsequently developed severe BMF [71]. Additional features of RS include intrauterine growth retardation, intracranial calcifications, developmental delay, fine sparse hair, nail dystrophy and other features that overlap with DC. The discovery of very short telomeres and TINF2 mutations in patients with DC proved that RS is a clinically severe variant of DC [53]. The specific diagnosis of RS requires the identification of exudative retinopathy, which must be distinguished from proliferative retinopathy, reported in non-RS DC [72].

\section{Coats plus/CRMCC}

The disorder known as Coats plus or CRMCC bears both clinical and etiological similarities to DC. Coats plus/CRMCC is characterized by bilateral exudative retinopathy, retinal telangiectasias, growth retardation, intracranial calcifications, bone abnormalities and gastrointestinal vascular ectasias. Some patients may also have dystrophic nails, sparse or graying hair and anemia. The clinical characteristics overlap with those of DC, and especially with RS, in which intracranial calcifications have also been reported [71]. Exome sequencing revealed that Coats plus is caused by compound heterozygous mutations in $C T C 1[8,9,44]$, a gene involved in telomere maintenance that, when mutated, can also cause DC (as mentioned earlier). Furthermore, like DC patients, Coats plus patients have telomeres that are below the first percentile for age; heterozygous mutation carriers have telomere lengths that are below average [8]. mia or squamous cell cancer. Identification of such patients is critical in order to optimize treatment modalities, since these individuals may have an underlying DC-related telomere biology disorder.

\section{Pulmonary fibrosis}

Idiopathic pulmonary fibrosis is a complex, multifactorial disease that leads to progressive lung scarring and fibrotic changes. The majority of cases are sporadic with a limited number of known environmental risk factors. Importantly, it is now known that a subset of cases of pulmonary fibrosis is due to an underlying inherited telomere biology disorder. Germline AD mutations in TERC and TERT have also been reported as a cause of pulmonary fibrosis in 5-10\% of cases who also have a family history of a telomere biology disorder, such as aplastic anemia or nonalcoholic/noninfectious liver disease. Short telomeres, in the absence of a known germline mutation, may also be a risk factor for the development of pulmonary fibrosis in the general population [73]. Large, population-based studies are required to further understand the connection between telomere biology and pulmonary fibrosis in individuals without an obvious family history.

\section{Nonalcoholic/noninfectious liver disease}

Patients with DC have been reported to have noncirrhotic portal hypertension, hepatic fibrosis and hepatopulmonary syndrome. The connection between the hepatic consequences of aberrant telomere biology was made in a study of individuals with apparently isolated liver disease due to germline mutations in TERT or TERC [12]. In that study, noninfectious, nonalcoholic liver disease was present as an isolated finding in individuals with mutations and relatives with BMF who had the same mutation. This 
suggests that a subset of these patients have an underlying telomere biology disorder and need to be treated as such.

\section{Clinical management of DC \& the related telomere biology disorders}

Evidence-based clinical care guidelines have not yet been developed for DC. However, our understanding of the clinical complications in DC is improving due to increased numbers of studies of DC.

\section{Genetic counseling \& testing}

It is important to identify the genetic cause of DC in families in order to begin to tailor medical management and to better understand disease etiology. Genetic education and counseling is extremely important for all individuals and families undergoing genetic testing for DC or any of the related telomere biology disorders. Individuals being tested should be given information on the clinical spectrum of the DC-related telomere biology disorders, on the modes of inheritance, and implications for the entire family. The identification of clinically affected and silent carriers of specific mutations has additional implications that must be addressed. Individuals undergoing testing need to understand that future medical complications cannot be predicted but they can be monitored.

Identification of the genetic causes of DC and the telomere biology disorders is also important for reproductive planning by individuals in families with these disorders. Carriers of a germline mutation may choose to have prenatal testing or to undergo preimplantation genetic diagnosis [74].

\section{Surveillance \& management}

There are no current, evidence-based data on screening strategies for patients with DC [75]. Consequently, the management of DC is based, in part, on the guidelines developed for FA [76]. FA is another rare cancer-prone IBMFS with similar complications, including BMF, cancer and developmental problems [77]. Regardless, each patient must have a clinical management plan tailored to his or her specific needs. Suggestions for clinical surveillance of patients with telomere biology disorders are shown in Table 2.

\section{Table 2. Clinical surveillance guidelines for individuals with dyskeratosis congenita.}

$\begin{array}{ll}\text { Problem } & \text { Suggested surveillance } \\ \text { BMF } & \text { Management depends on the severity. If CBCs are normal, consider } \\ & \text { an annual CBC to identify trends and early manifestations } \\ & \text { Baseline bone marrow aspiration and biopsy with careful } \\ & \text { morphologic examination and cytogenetic studies. Consider yearly } \\ \text { bone marrow evaluation }\end{array}$

$\mathrm{CBCs}$ and bone marrow evaluation should be obtained more frequently if cytopenias are present and at the discretion of the primary hematologist

BMF - patients on Special monitoring is required for patients on androgens for BMF androgens

Check liver function tests prior to starting and then every 3 months

Perform liver ultrasound examination prior to initiation and semiannually for adenomas, carcinomas or fibrosis

Check cholesterol and triglycerides prior to starting and every 6 months

Carefully follow growth and obtain baseline bone age in pediatric patients. Consider endocrinology evaluation

Cancer Most solid tumors develop after the first decade of life. Patient should be taught how to perform a monthly self-examination for oral, head and neck cancer

Annual cancer screening by a dentist and an otolaryngologist. Follow oral leukoplakia carefully and biopsy any changes or suspicious sites

Annual gynecologic evaluation for females

Annual dermatologic evaluation

Pulmonary fibrosis Annual pulmonary function tests are recommended at diagnosis or at an age when the patient can properly perform the test. Early evaluation for shortness of breath or unexplained cough

Counsel patients to stop smoking, if applicable

Dental and otolaryngology

Dental hygiene and screening every 6 months

Maintain good oral hygiene

Inform the primary dentist of the patient's increased risk of oral, head and neck squamous cell cancers

Carefully monitor oral leukoplakia and biopsy suspicious lesions early

Ophthalmic

Annual (or more frequent, if needed) examination to detect/correct vision problems, abnormally growing eyelashes, blocked tear ducts and to look for retinal changes, bleeding, cataracts and glaucoma

Endocrinology Baseline bone density scan to evaluate for osteopenia. Follow-up bone density scans yearly or as recommended by physician

Careful monitoring of growth and evaluation as needed

Development Thorough evaluation for developmental delay and therapy/support, as needed

Gastrointestinal Evaluate for clinical history suspicious for esophageal stenosis and hepatic and/or enteropathy and refer as needed. Obtain baseline liver function tests and use caution when administering potential hepatotoxic medications

These are guidelines only. The primary treatment team should tailor a plan to address each patient's specific need(s).

BMF: Bone marrow failure; CBC: Complete blood count. 


\section{Bone marrow failure}

Clinically significant cytopenias are a major problem for patients with DC and the related telomere biology disorders. In many instances, BMF may be the presenting feature of this spectrum of illnesses. All patients with new-onset BMF should first be evaluated for FA by chromosome breakage analysis. If that test is normal, clinical telomere length testing should be performed using peripheral blood leukocytes [64].

The FA consensus guidelines are often used for DC since the complications in the disorders are somewhat similar. As in FA, BMF in patients with DC does not respond to immunosuppressive medications [78], leaving allogeneic hematopoietic stem cell transplant (HSCT), the only opportunity to cure the bone marrow defect. Data on the outcome after HSCT in patients with DC are sparse, and consist primarily of case reports or small case series [79-87]. The outcomes have generally been poor, with complications including graft failure, pulmonary fibrosis, liver disease and graft-versus-host disease. Consequently, HSCT for DC should be performed at centers experienced with this rare disorder. Reduced-intensity regimens are being studied at a few institutions in order to improve long-term outcomes.

It is very important to test potential related bone marrow donors prior to HSCT because of the clinical heterogeneity of DC and the presence of silent carriers. For example, there are two case reports of a related HSCT donor being identified as a mutation carrier only after either failure to engraft or failure to mobilize stem cells for their relative with DC. This resulted in failure of the HSCT and death of the patients [88,89].

Androgens may be considered in patients with severe BMF who do not have a suitable HSCT donor or who are not eligible for HSCT. Oxymethalone is the most commonly used androgen in DC-related BMF, but several investigators are studying danazol in this setting. Approximately half of patients with DC appear to respond to androgens and no longer require red blood cell or platelet transfusion support [90]. However, patients with DC may be more sensitive to androgen-related side effects, such as poor liver function, abnormal lipid and cholesterol levels and risk of liver adenomas. Hematopoietic growth factors may be useful in BMF. Unfortunately, splenic peliosis and splenic rupture were reported in two individuals with DC who received the combination of androgens and G-CSF [91]. G-CSF with erythropoietin has occasionally been useful but should not be used in combination with androgens.

\section{Pulmonary fibrosis}

Patients with DC are at high risk of pulmonary fibrosis. Pulmonary fibrosis may be the presenting sign of a DC-related telomere biology disorder or it may develop at any time. Notably, in individuals with TERT or TERC mutations, pulmonary fibrosis, even in the elderly, may be the presenting feature in a family. There are no genotype-phenotype data on the relationship between specific causative genes and pulmonary fibrosis in patients with classic DC. Pulmonary fibrosis appears to occur at an earlier age in DC patients after HSCT compared with patients with DC who did not undergo HSCT [92]. However, this observation is probably multifactorial. Patients with DC who undergo HSCT may be more clinically severe than those who do not and the HSCT itself can increase the risk of pulmonary fibrosis. Lung transplantation for pulmonary fibrosis after HSCT has been successfully reported in one patient with DC. Other than lung transplantation, the management of telomere biology disorder-related pulmonary fibrosis is primarily supportive with administration of supplemental oxygen.

\section{Expert commentary}

The last decade of basic science and clinical studies has greatly improved our knowledge of the etiology of DC and of telomere biology. Understanding of the molecular aspects of telomere function and regulation has advanced, in part, due to study of the defects identified in DC. However, much more remains unclear.

Detailed phenotypic studies of all patients with telomere biology disorders, including DC and the related disorders, and their family members are critical to improve our understanding of the clinical consequences of this disorder. The spectrum of the clinical features associated with DC-related telomere biology disorders is growing and the clinical community requires improved understanding of this overlap. Proper diagnosis of these complex disorders is especially important in order to perform appropriate cancer screening and genetic counseling.

Ongoing studies utilizing whole exome and whole genome sequencing are underway in all of the disorders described herein. In the very near future, the underlying genetic causes of the telomere biology disorders will be known, leading to improved understanding of the underlying molecular biology. The major goal of all of these studies should be to improve clinical outcomes. Therapeutics aimed at lengthening or stabilizing telomeres in patients with DC have the theoretical potential to improve outcomes, but there are none currently being studied in humans. Multicenter studies of the optimal HSCT regimen for DC are required in order to develop the regimen with the least toxicity and best long-term outcomes. As with any rare disease, scientific collaboration between investigators around the world and from many disciplines is crucial to advance understanding of DC etiology and to improve outcomes.

\section{Five-year view}

In the next 5 years the authors predict that improvements in HSCT for DC will be developed, the majority of families will know the genetic etiology of their disease, a better appreciation of the clinical and genetic overlap between the telomere biology disorders will be attained, exploration of genetic and environmental modifiers may begin to elucidate the underlying mechanisms of disease heterogeneity and development of targeted therapeutics will begin.

\section{Financial \& competing interests disclosure}

The authors have no relevant affiliations or financial involvement with any organization or entity with a financial interest in or financial conflict with the subject matter or materials discussed in the manuscript. This includes employment, consultancies, honoraria, stock ownership or options, expert testimony, grants or patents received or pending, or royalties.

No writing assistance was utilized in the production of this manuscript. 


\section{Key issues}

- Dyskeratosis congenita (DC) is clinically and genetically heterogeneous.

- Individuals with aplastic anemia, pulmonary fibrosis or liver disease may have a telomere biology disorder that biologically overlaps with DC.

- DC, Hoyeraal-Hreidarsson syndrome, Revesz syndrome and Coats plus/cerebroretinal microangiopathy with calcifications and cysts are clinically and biologically related.

- Patients with telomere biology disorders may be at increased risk of cancer and bone marrow failure.

- Other than hematopoietic stem cell transplantation for bone marrow failure and lung transplantation for pulmonary fibrosis, there are no durative therapeutic modalities.

\section{References}

1 Heiss NS, Knight SW, Vulliamy TJ et al. $\mathrm{X}$-linked dyskeratosis congenita is caused by mutations in a highly conserved gene with putative nucleolar functions. Nat. Genet. 19(1), 32-38 (1998).

2 Mitchell JR, Wood E, Collins K. A telomerase component is defective in the human disease dyskeratosis congenita. Nature 402(6761), 551-555 (1999).

3 Vulliamy T, Marrone A, Goldman F et al. The RNA component of telomerase is mutated in autosomal dominant dyskeratosis congenita. Nature 413(6854), 432-435 (2001).

4 Vulliamy TJ, Walne A, Baskaradas A, Mason PJ, Marrone A, Dokal I. Mutations in the reverse transcriptase component of telomerase (TERT) in patients with bone marrow failure. Blood Cells Mol. Dis. 34(3), 257-263 (2005).

5 Vulliamy TJ, Marrone A, Knight SW, Walne A, Mason PJ, Dokal I. Mutations in dyskeratosis congenita: their impact on telomere length and the diversity of clinical presentation. Blood 107(7), 2680-2685 (2006).

6 Alter BP, Giri N, Savage SA, Rosenberg PS. Cancer in dyskeratosis congenita. Blood 113(26), 6549-6557 (2009).

7 Alter BP, Baerlocher GM, Savage SA et al. Very short telomere length by flow fluorescence in situ hybridization identifies patients with dyskeratosis congenita. Blood 110(5), 1439-1447 (2007).

8 Anderson BH, Kasher PR, Mayer J et al. Mutations in CTC1, encoding conserved telomere maintenance component 1, cause Coats plus. Nat. Genet. 44(3), 338-342 (2012).

9 Polvi A, Linnankivi T, Kivelä T et al. Mutations in CTC1, encoding the CTS telomere maintenance complex component 1, cause cerebroretinal microangiopathy with calcifications and cysts. Am. J. Hum. Genet. 90(3), 540-549 (2012).
10 Tsakiri KD, Cronkhite JT, Kuan PJ et al. Adult-onset pulmonary fibrosis caused by mutations in telomerase. Proc. Natl Acad. Sci. USA 104(18), 7552-7557 (2007).

11 Armanios MY, Chen JJ, Cogan JD et al. Telomerase mutations in families with idiopathic pulmonary fibrosis. N. Engl. J. Med. 356(13), 1317-1326 (2007).

12 Calado RT, Regal JA, Kleiner DE et al. A spectrum of severe familial liver disorders associate with telomerase mutations. PLoS ONE 4(11), e7926 (2009).

13 Meyne J, Ratliff RL, Moyzis RK. Conservation of the human telomere sequence (TTAGGG)n among vertebrates. Proc. Natl Acad. Sci. USA 86(18), 7049-7053 (1989).

14 de Lange T, Shiue L, Myers RM et al. Structure and variability of human chromosome ends. Mol. Cell. Biol. 10(2), 518-527 (1990).

15 Wright WE, Tesmer VM, Huffman KE, Levene SD, Shay JW. Normal human chromosomes have long G-rich telomeric overhangs at one end. Genes Dev. 11(21), 2801-2809 (1997).

16 Olovnikov AM. A theory of marginotomy. The incomplete copying of template margin in enzymic synthesis of polynucleotides and biological significance of the phenomenon. J. Theor. Biol. 41(1), 181-190 (1973).

17 Watson JD. Origin of concatemeric T7 DNA. Nature New Biol. 239(94), 197-201 (1972).

18 Hayflick L, Moorhead PS. The serial cultivation of human diploid cell strains. Exp. Cell Res. 25, 585-621 (1961).

19 Lundblad V, Szostak JW. A mutant with a defect in telomere elongation leads to senescence in yeast. Cell 57(4), 633-643 (1989).

20 Zou Y, Sfeir A, Gryaznov SM, Shay JW, Wright WE. Does a sentinel or a subset of short telomeres determine replicative senescence? Mol. Biol. Cell 15(8), 3709-3718 (2004).
21 Liti G, Louis EJ. NEJ1 prevents NHEJdependent telomere fusions in yeast without telomerase. Mol. Cell 11(5), 1373-1378 (2003).

22 Mieczkowski PA, Mieczkowska JO, Dominska M, Petes TD. Genetic regulation of telomere-telomere fusions in the yeast Saccharomyces cerevisae. Proc. Natl Acad. Sci. USA 100(19), 10854-10859 (2003).

23 McClintock B. The stability of broken ends of chromosomes in Zea mays. Genetics 26(2), 234-282 (1941).

24 Hanahan D. Benefits of bad telomeres. Nature 406(6796), 573-574 (2000).

25 Nugent CI, Lundblad V. The telomerase reverse transcriptase: components and regulation. Genes Dev. 12(8), 1073-1085 (1998).

26 Blackburn EH. Telomeres and telomerase: their mechanisms of action and the effects of altering their functions. FEBS Lett. 579(4), 859-862 (2005).

27 Hamma T, Ferré-D'Amaré AR. The box H/ACA ribonucleoprotein complex: interplay of RNA and protein structures in post-transcriptional RNA modification. J. Biol. Chem. 285(2), 805-809 (2010).

28 Trahan C, Martel C, Dragon F. Effects of dyskeratosis congenita mutations in dyskerin, NHP2 and NOP10 on assembly of H/ACA pre-RNPs. Hum. Mol. Genet. 19(5), 825-836 (2010).

29 Venteicher AS, Abreu EB, Meng Z et al. A human telomerase holoenzyme protein required for Cajal body localization and telomere synthesis. Science 323(5914), 644-648 (2009).

30 Grozdanov PN, Roy S, Kittur N, Meier UT. SHQ1 is required prior to NAF1 for assembly of H/ACA small nucleolar and telomerase RNPs. RNA 15(6), 1188-1197 (2009).

31 Venteicher AS, Meng Z, Mason PJ, Veenstra TD, Artandi SE. Identification of ATPases pontin and reptin as telomerase 
components essential for holoenzyme assembly. Cell 132(6), 945-957 (2008).

32 Fatica A, Dlakic M, Tollervey D. Naf1 p is a box H/ACA snoRNP assembly factor. RNA 8(12), 1502-1514 (2002).

33 Ulaner GA, Hu JF, Vu TH, Giudice LC, Hoffman AR. Telomerase activity in human development is regulated by human telomerase reverse transcriptase (hTERT) transcription and by alternate splicing of hTERT transcripts. Cancer Res. 58(18), 4168-4172 (1998).

34 Härle-Bachor C, Boukamp P. Telomerase activity in the regenerative basal layer of the epidermis inhuman skin and in immortal and carcinoma-derived skin keratinocytes. Proc. Natl Acad. Sci. USA 93(13), 6476-6481 (1996).

35 Hiyama K, Hirai Y, Kyoizumi S et al. Activation of telomerase in human lymphocytes and hematopoietic progenitor cells. J. Immunol. 155(8), 3711-3715 (1995).

36 Chiu CP, Dragowska W, Kim NW et al. Differential expression of telomerase activity in hematopoietic progenitors from adult human bone marrow. Stem Cells 14(2), 239-248 (1996).

37 Armanios M. Syndromes of telomere shortening. Annu. Rev. Genomics Hum. Genet. 10, 45-61 (2009).

38 Savage SA, Bertuch AA. The genetics and clinical manifestations of telomere biology disorders. Genet. Med. 12(12), 753-764 (2010).

39 Nelson ND, Bertuch AA. Dyskeratosis congenita as a disorder of telomere maintenance. Mutat. Res. 730(1-2), 43-51 (2012).

40 Yamaguchi H, Calado RT, Ly H et al. Mutations in TERT, the gene for telomerase reverse transcriptase, in aplastic anemia. N. Engl. J. Med. 352(14), 1413-1424 (2005).

41 Aalbers AM, Kajigaya S, van den HeuvelEibrink MM, van der Velden VH, Calado RT, Young NS. Human telomere disease due to disruption of the CCAAT box of the TERC promoter. Blood 119(13), 3060-3063 (2012).

42 Zhong F, Savage SA, Shkreli M et al. Disruption of telomerase trafficking by TCAB1 mutation causes dyskeratosis congenita. Genes Dev. 25(1), 11-16 (2011).

43 Canudas S, Houghtaling BR, Bhanot M et al. A role for heterochromatin protein $1 \gamma$ at human telomeres. Genes Dev. 25(17), 1807-1819 (2011).
44 Savage SA. Connecting complex disorders through biology. Nat. Genet. 44(3), 238-240 (2012).

45 Keller RB, Gagne KE, Usmani GN et al. CTC1 mutations in a patient with dyskeratosis congenita. Pediatr. Blood Cancer 59(2), 311-314 (2012).

46 Walne AJ, Bhagat T, Kirwan M et al. Mutations in the telomere capping complex in bone marrow failure and related syndromes. Haematologica 98(3), 334-338 (2013).

47 Ballew BJ, Yeager M, Jacobs K et al. Germline mutations of regulator of telomere elongation helicase 1, RTEL1, in Dyskeratosis congenita. Hum. Genet. 132(4), 473-480 (2013).

48 Uringa EJ, Lisaingo K, Pickett HA et al. RTEL1 contributes to DNA replication and repair and telomere maintenance. Mol. Biol. Cell 23(14), 2782-2792 (2012).

49 Vannier JB, Pavicic-Kaltenbrunner V, Petalcorin MI, Ding H, Boulton SJ. RTEL1 dismantles T loops and counteracts telomeric G4-DNA to maintain telomere integrity. Cell 149(4), 795-806 (2012).

50 Walne AJ, Vulliamy T, Kirwan M, Plagnol V, Dokal I. Constitutional mutations in RTEL1 cause severe dyskeratosis congenita. Am. J. Hum. Genet. 92(3), 448-453 (2013).

51 Touzot F, Callebaut I, Soulier J et al. Function of Apollo (SNM1B) at telomere highlighted by a splice variant identified in a patient with Hoyeraal-Hreidarsson syndrome. Proc. Natl Acad. Sci. USA 107(22), 10097-10102 (2010).

52 Walne AJ, Vulliamy T, Beswick R, Kirwan M, Dokal I. Mutations in C16orf57 and normal-length telomeres unify a subset of patients with dyskeratosis congenita, poikiloderma with neutropenia and Rothmund-Thomson syndrome. Hum. Mol. Genet. 19(22), 4453-4461 (2010).

53 Savage SA, Giri N, Baerlocher GM, Orr N, Lansdorp PM, Alter BP. TINF2, a component of the shelterin telomere protection complex, is mutated in dyskeratosis congenita. Am. J. Hum. Genet. 82(2), 501-509 (2008).

54 Vulliamy T, Marrone A, Szydlo R, Walne A, Mason PJ, Dokal I. Disease anticipation is associated with progressive telomere shortening in families with dyskeratosis congenita due to mutations in TERC. Nat. Genet. 36(5), 447-449 (2004).

55 Armanios M, Chen JL, Chang YP et al. Haploinsufficiency of telomerase reverse transcriptase leads to anticipation in autosomal dominant dyskeratosis congenita. Proc. Natl Acad. Sci. USA 102(44), 15960-15964 (2005).

56 Goldman F, Bouarich R, Kulkarni S et al. The effect of TERC haploinsufficiency on the inheritance of telomere length. Proc. Natl Acad. Sci. USA 102(47), 17119-17124 (2005).

57 Jongmans MC, Verwiel ET, Heijdra Y et al. Revertant somatic mosaicism by mitotic recombination in dyskeratosis congenita. Am. J. Hum. Genet. 90(3), 426-433 (2012).

58 Sorrow JM Jr, Hitch JM. Dyskeratosis congenita. First report of its occurrence in a female and a review of the literature. Arch. Dermatol. 88, 340-347 (1963).

59 Vulliamy TJ, Kirwan MJ, Beswick R et al. Differences in disease severity but similar telomere lengths in genetic subgroups of patients with telomerase and shelterin mutations. PLoS ONE 6(9), e24383 (2011).

60 Atkinson JC, Harvey KE, Domingo DL et al. Oral and dental phenotype of dyskeratosis congenita. Oral Dis. 14(5), 419-427 (2008).

61 Tsilou ET, Giri N, Weinstein S, Mueller C, Savage SA, Alter BP. Ocular and orbital manifestations of the inherited bone marrow failure syndromes: Fanconi anemia and dyskeratosis congenita. Ophthalmology 117(3), 615-622 (2010).

62 Rackley S, Pao M, Seratti GF et al. Neuropsychiatric conditions among patients with dyskeratosis congenita: a link with telomere biology? Psychosomatics 53(3), 230-235 (2012).

63 Gadalla SM, Cawthon R, Giri N, Alter BP, Savage SA. Telomere length in blood, buccal cells, and fibroblasts from patients with inherited bone marrow failure syndromes. Aging (Albany. NY) 2(11), 867-874 (2010)

64 Alter BP, Rosenberg PS, Giri N, Baerlocher GM, Lansdorp PM, Savage SA. Telomere length is associated with disease severity and declines with age in dyskeratosis congenita. Haematologica 97(3), 353-359 (2012).

65 Lin KW, Yan J. The telomere length dynamic and methods of its assessment. J. Cell. Mol. Med. 9(4), 977-989 (2005).

66 Baird DM. New developments in telomere length analysis. Exp. Gerontol. 40(5), 363-368 (2005).

67 Walne AJ, Vulliamy T, Beswick R, Kirwan M, Dokal I. TINF2 mutations result in very short telomeres: analysis of a large cohort of patients with dyskeratosis 
congenita and related bone marrow failure syndromes. Blood 112(9), 3594-3600 (2008).

68 Hoyeraal HM, Lamvik J, Moe PJ. Congenital hypoplastic thrombocytopenia and cerebral malformations in two brothers. Acta Paediatr. Scand. 59(2), 185-191 (1970).

69 Hreidarsson S, Kristjansson K, Johannesson G, Johannsson JH. A syndrome of progressive pancytopenia with microcephaly, cerebellar hypoplasia and growth failure. Acta Paediatr. Scand. 77(5), 773-775 (1988).

70 Knight SW, Heiss NS, Vulliamy TJ et al. Unexplained aplastic anaemia, immunodeficiency, and cerebellar hypoplasia (Hoyeraal-Hreidarsson syndrome) due to mutations in the dyskeratosis congenita gene, DKC1. Br. J. Haematol. 107(2), 335-339 (1999).

71 Revesz T, Fletcher S, al-Gazali LI, DeBuse P. Bilateral retinopathy, aplastic anaemia, and central nervous system abnormalities: a new syndrome? J. Med. Genet. 29(9), 673-675 (1992).

72 Mason JO 3rd, Yunker JJ, Nixon PA et al. Proliferative retinopathy as a complication of aplastic anemia and dyskeratosis congenita. Retinal Cases \& Brief Reports 3 , 259-262 (2009).

73 Alder JK, Chen JJ, Lancaster L et al. Short telomeres are a risk factor for idiopathic pulmonary fibrosis. Proc. Natl Acad. Sci. USA 105(35), 13051-13056 (2008).

74 Savage SA, Alter BP. Dyskeratosis congenita. Hematol. Oncol. Clin. North Am. 23(2), 215-231 (2009).

75 Savage SA, Dokal I, Armanios M et al. Dyskeratosis congenita: the first NIH clinical research workshop. Pediatr. Blood Cancer 53(3), 520-523 (2009).

76 Eiler ME, Frohnmayer D, Frohnmayer L, Larsen K, Owen J (Eds). Fanconi Anemia: Guidelines for Diagnosis and Management (3rd Edition). Fanconi Anemia Research Fund, Inc., OR, USA (2008).
Shimamura A, Alter BP. Pathophysiology and management of inherited bone marrow failure syndromes. Blood Rev. 24(3), 101-122 (2010).

78 Al-Rahawan MM, Giri N, Alter BP. Intensive immunosuppression therapy for aplastic anemia associated with dyskeratosis congenita. Int. J. Hematol. 83(3), 275-276 (2006).

79 Berthou C, Devergie A, D'Agay MF et al. Late vascular complications after bone marrow transplantation for dyskeratosis congenita. Br. J. Haematol. 79(2), 335-336 (1991).

80 De La Fuente J, Dokal I. Dyskeratosis congenita: advances in the understanding of the telomerase defect and the role of stem cell transplantation. Pediatric Transplantation 11(6), 584-594 (2007).

81 Yabe M, Yabe H, Hattori K et al. Fatal interstitial pulmonary disease in a patient with dyskeratosis congenita after allogeneic bone marrow transplantation. Bone Marrow Transplant. 19(4), 389-392 (1997).

82 Dror Y, Freedman MH, Leaker $M$ et al. Low-intensity hematopoietic stem-cell transplantation across human leucocyte antigen barriers in dyskeratosis congenita. Bone Marrow Transplant. 31(10), 847-850 (2003).

83 Brazzola P, Duval M, Fournet JC et al. Fatal diffuse capillaritis after hematopoietic stem-cell transplantation for dyskeratosis congenita despite low-intensity conditioning regimen. Bone Marrow Transplant. 36(12), 1103-1105; author reply 1105 (2005).

84 Ostronoff F, Ostronoff M, Calixto R et al. Fludarabine, cyclophosphamide, and antithymocyte globulin for a patient with dyskeratosis congenita and severe bone marrow failure. Biol. Blood Marrow Transplant. 13(3), 366-368 (2007).

85 Vuong LG, Hemmati PG, Neuburger $S$ et al. Reduced-intensity conditioning using fludarabine and antithymocyte globulin alone allows stable engraftment in a patient with dyskeratosis congenita. Acta Haematol. 124(4), 200-203 (2010).
86 Dietz AC, Orchard PJ, Baker KS et al. Disease-specific hematopoietic cell transplantation: nonmyeloablative conditioning regimen for dyskeratosis congenita. Bone Marrow Transplant. 46(1), 98-104 (2011)

87 Nishio N, Takahashi Y, Ohashi $\mathrm{H}$ et al. Reduced-intensity conditioning for alternative donor hematopoietic stem cell transplantation in patients with dyskeratosis congenita. Pediatr. Transplant. 15(2), 161-166 (2011).

88 Fogarty PF, Yamaguchi H, Wiestner A et al. Late presentation of dyskeratosis congenita as apparently acquired aplastic anaemia due to mutations in telomerase RNA. Lancet 362 (9396), 1628-1630 (2003).

89 Denny CC, Wilfond BS, Peters JA, Giri N, Alter BP. All in the family: disclosure of 'unwanted' information to an adolescent to benefit a relative. Am. J. Med. Genet. A 146A(21), 2719-2724 (2008).

90 Khincha P, Wentzensen I, Giri N, Alter BP, Savage SA. Response to androgen therapy and side effects in patients with dyskeratosis congenita. Presented at: American Society of Hematology Annual Meeting Atlanta, GA, USA, 6-11 December 2012.

91 Giri N, Pitel PA, Green D, Alter BP. Splenic peliosis and rupture in patients with dyskeratosis congenita on androgens and granulocyte colony-stimulating factor. Br. J. Haematol. 138(6), 815-817 (2007).

92 Giri N, Lee R, Faro A et al. Lung transplantation for pulmonary fibrosis in dyskeratosis congenita: case report and systematic literature review. BMC Blood Disord. 11, 3 (2011).

93 Knight S, Vulliamy T, Copplestone A, Gluckman E, Mason P, Dokal I. Dyskeratosis Congenita (DC) Registry: identification of new features of DC. $\mathrm{Br}$. J. Haematol. 103(4), 990-996 (1998).

94 Vulliamy T, Dokal I. Dyskeratosis congenita. Semin. Hematol. 43(3), 157-166 (2006). 